\title{
High-resolution air quality modelling and time scale analysis of ozone and NOx in Osaka, Japan
}

\author{
K. L. Shrestha, A. Kondo, A. Kaga \& Y. Inoue \\ Graduate School of Engineering, Osaka University, Japan
}

\begin{abstract}
The ozone pollution in the Osaka region is mainly influenced by the long-range transport of tropospheric ozone or its precursors from outside in winter but nearly $50 \%$ of the total ozone is chemically produced by regional emissions in summertime. The effects of the regional transport and the chemical production of ozone by regional emissions in summertime can be analysed by spectral decomposition of the time series and studying the inherent intra-day, diurnal and longer-term time scales in the time series of ozone and NOx. The relative contributions of these time scale components associated with different physical processes are also used to evaluate the performance of air quality models in simulating ozone and NOx. We used the Japanese national emission data with resolution of $10-\mathrm{km}$ and $1-\mathrm{km}$ resolution in the MM5-CMAQ modelling system for the nested simulation in Osaka with the finest domain having 1-km grid size. Among the different time scales, diurnal time scale has the highest correlation for ozone, and longer time scale has the highest correlation for NO and NO2. Spatial variation in the simulation of NOx is evident from the study of correlation of diurnal and longer-term time scales, with higher correlations in the longer-term time scale. Overall, the time scale analysis of ozone and NOx time series has proved useful for detailed quantitative analysis of the air quality modelling in Osaka.
\end{abstract}

Keywords: time scale analysis, air quality evaluation, urban air quality, MM5, CMAQ.

\section{Introduction}

Since the ozone concentration is affected by cyclic patterns of meteorological forcings as well as the anthropogenic influences such as traffic emission of $\mathrm{NO}_{x}$ 
and VOCs [1], the study of time scale components can be used to analyze such influences. There are different components of time scales used in the analysis of meteorological variables, ozone and its precursors. The highest and lowest resolvable frequencies are determined by the sampling interval and the length of the data record [2]. Since our simulation time period is one-month, we can only resolve time scales of about 10 days. Considering this limitation, only the intra-day scale, diurnal scale and longer-term scale have been used in our study. The intra-day scale has frequencies less than 12 hours, diurnal scale has frequencies around 24 hours and longer-term scale contains all the frequencies above the diurnal scale. The frequency bands were determined [2,3], and the clean separation of the intraday, diurnal and longer-term scales was confirmed from the periodograms using the Fast Fourier Transform (FFT) technique (not shown here). The KolmogorovZurbenko (KZ) filter method is a widely used technique for the separation of different time scales of meteorological variables [4]. Besides having a powerful and efficient separation characteristics, KZ filter method can be applied to datasets having missing observation data as well. This method has also been successfully used for the analysis of different scales of ozone time series data so that the temporal information hidden in the time series data can be extracted cleanly by separating the time series into different scales of motion $[1,3,5]$. In order to determine the time scale frequencies, logarithmic scale was used for ozone and NOx while the meteorological data were analyzed without using the logarithmic scale [2,3]. This work presents the results of time scale analysis of ozone, and Nox in Osaka region for July, 2002.

\subsection{Domain and grid structures}

The Osaka prefecture region was selected as the target area for evaluating the MM5-CMAQ modelling system (Fig. 1). The area around Osaka was used as the finest domain area with a grid size of $1 \mathrm{~km}$ (Domain 3). This domain was nested inside a coarser domain with a grid size of $3 \mathrm{~km}$ (Domain 2). These domains were further nested in the coarsest domain of $9 \mathrm{~km}$ grid size covering nearly all of Japan (Domain 1).

\subsection{Meteorological modelling}

The Pennsylvania State University/National Center for Atmospheric Research Mesoscale Modelling System (MM5) is a limited-area, non-hydrostatic, terrainfollowing sigma coordinate model designed to simulate or predict mesoscale and regional-scale atmospheric circulation $[6,7]$. In this study, the national mesoscale grid point value data (GPV-MSM) provided by Japan Meteorological Agency was used to initialize the MM5 model. The grid size of each pressure level of this data is $0.2^{\circ} \times 0.25^{\circ}$. The simple ice microphysics, Dudhia's longwave and shortwave radiation scheme, Grell cumulus parameterization scheme and Medium-Range Forecast (MRF) PBL scheme [8] with multi-layer soil model [9] were used in MM5 simulation. 


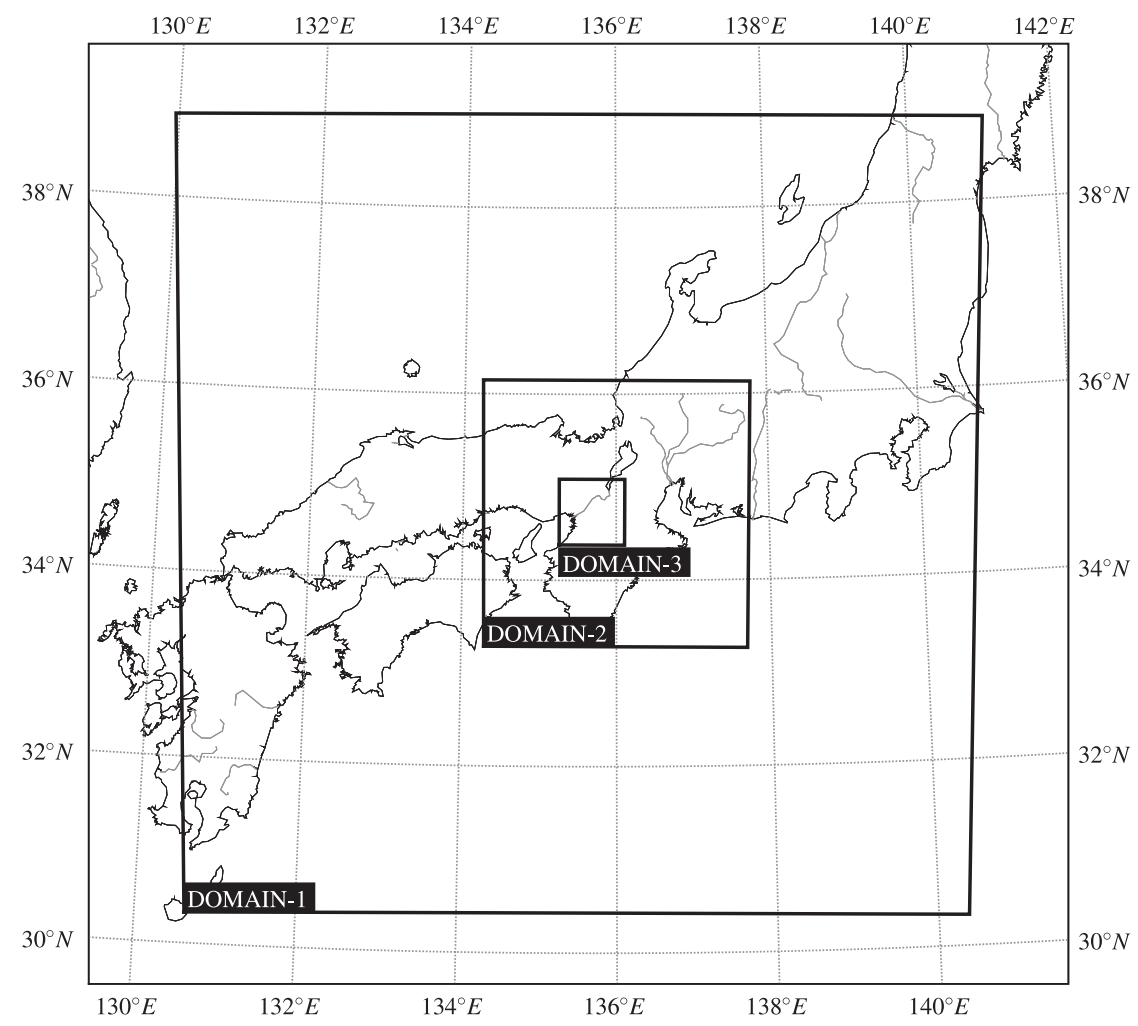

Figure 1: Nesting of model domains for CMAQ.

\subsection{Emission modelling}

The emission source data was obtained from Japan Clean Air Program (JCAP). For the 9-km domain (Domain 1) and 3-km domain (Domain 2), the national emission data with the spatial resolution of $0.125^{\circ}$ by $0.0833^{\circ}$ was used. For $1-\mathrm{km}$ domain (Domain 3), the data covering the Kansai region with $0.0125^{\circ}$ by $0.00833^{\circ}$ resolution was used. This emission data is available as the average data for the month of July. Another feature of this data is the availability of weekday and weekend mobile emission data. Similarly, the biogenic emission has also been incorporated as the average of July.

\subsection{CMAQ modelling}

Community Multi-scale Air Quality (CMAQ) model is a Eulerian-type air quality model that simulates concurrently the atmospheric and land processes affecting the transport, transformation, and deposition of air pollutants and their precursors, on both regional and urban scales [10]. CMAQ is configured with the chemical 
mechanism of Statewide Air Pollution Research Center (SAPRC-99) mechanism, which is a detailed mechanism for the gas-phase atmospheric reactions of volatile organic compounds (VOCs) and oxides of nitrogen (NOx) in urban and regional atmospheres.

\section{Results and discussion}

\subsection{Time scale analysis}

For ozone as well as meteorological variables, the relative contribution of the different temporal components to the total time series can be examined from the variances of the components [11]. It can assist in examining the accuracy of the model in predicting the individual time scale components that are associated with different physical processes. For evaluation of the simulated meteorological and air quality variables, the observation data were obtained from the "Air Pollution Continuous Monitoring Network Data Files" provided by Environmental Pollution Control Center, Osaka Prefecture.The following results were obtained from simulation results of Domain 3 (1-km grid) of MM5-CMAQ system.

Figure 2 shows the contribution of different time scale components of time series of near-surface air temperature and wind speed using the percentage contribution of variance of each component to the total variance. The contributions of intra-day, diurnal and longer-term components are differing from the observed values by $0.95,1.01$ and 0.99 times respectively for the temperature time series and by $0.88,1.07$ and 0.97 times respectively for the wind speed time series. It indicates a strong diurnal influence on air temperature with the intra-day component differing the most from the observed values. Though one would expect the intra-day component to have larger underpredictions than this result [11], the use of very high resolution $(1-\mathrm{km})$ grid and high resolution meteorological analysis data could have been one of the factors behind this improvement. The wind speed is also being influenced by diurnal forcings but the contribution of intra-day component is nearly 6 times than that of air temperature. Ohsawa et al. [12] reported that the maximum wind speed due to southerly sea breeze are underpredicted by MM5 even in fine domains of nearly $1 \mathrm{~km}$ grid size and that a better representation of diurnal variation is necessary in the summertime in regions with complex terrain. This situation is improved in our results because four dimensional data assimilation (FDDA) is not used and so a significant loss in the contribution of diurnal variations is avoided. In addition, the contribution of intra-day component has increased due to the absence of smoothing of intra-day variations by FDDA, and the local wind circulations being captured at very fine resolution of $1 \mathrm{~km}$.

The contribution of intra-day, diurnal and longer-term components are differing from the observed values by $2.95,0.84$ and 1.05 times respectively for ozone, 1.69, 1.80 and 0.66 times respectively for $\mathrm{NO}_{2}$, and $0.99,1.91$ and 0.38 times respectively for NO time series (Fig. 3). The intra-day component of ozone has the highest difference in contribution (nearly 3 times) when compared with the observed data, and this might have contributed to the slightly less contribution in diurnal 
Simulated

(a) Temperature

\section{Observed}
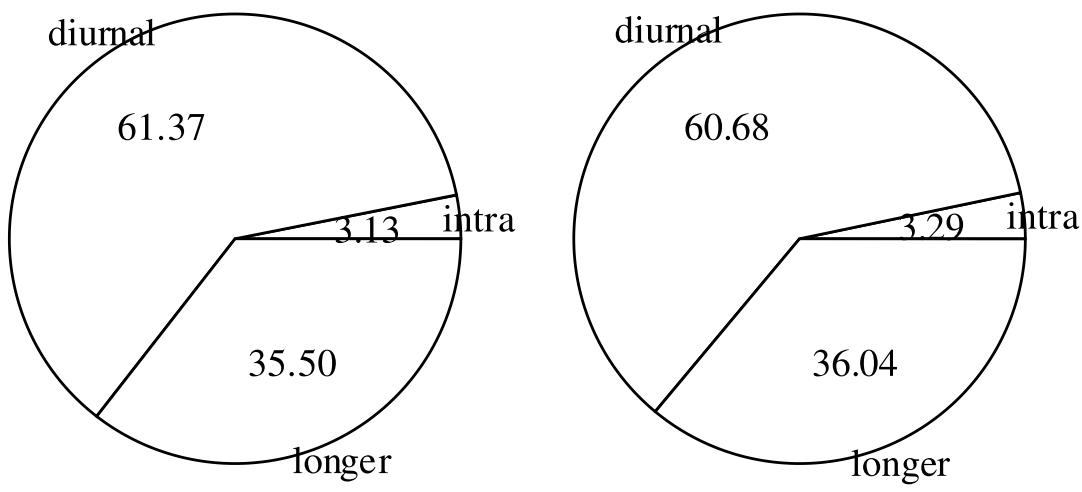

Simulated

(b) Wind speed
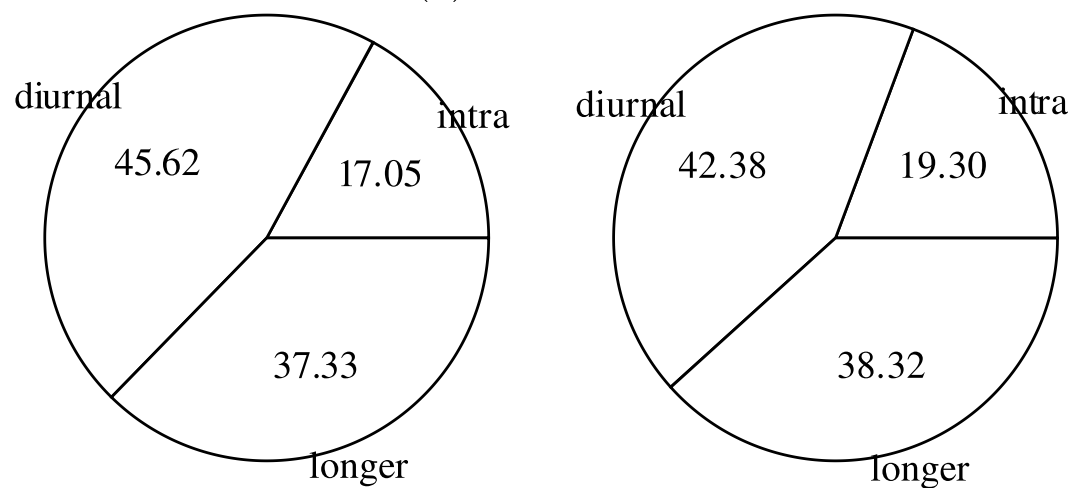

Figure 2: Contribution of different time scales to the total variance of (a) nearsurface air temperature and (b) wind speed. The values are shown as percentage of contribution of time scale component to the total variance.

component. The overestimation of intra-day component of ozone may be partially attributed to the overestimation of the intra-day component of its precursors, and also to the fact that the intra-day fluctuations of ozone are significantly affected by the intra-day wind speed fluctuations. Moreover, the uncertainties in the local emissions of ozone precursors also affect the intra-day fluctuations of ozone. The intra-day component is well-captured by MM5-CMAQ system for NO than its longer-term and diurnal components. For $\mathrm{NO}_{2}$, the results show overpredictions in the contribution of variability from intra-day and diurnal components and under- 
Simulated

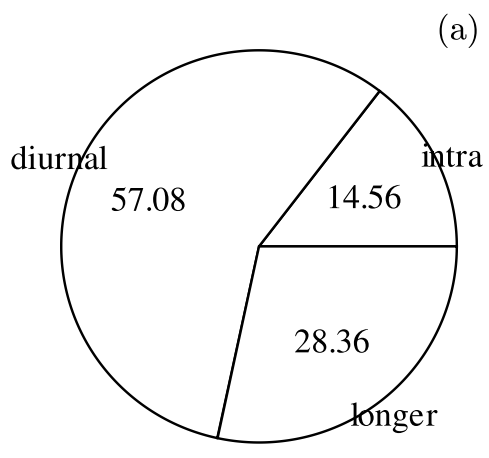

Simulated
Observed

(a) Ozone

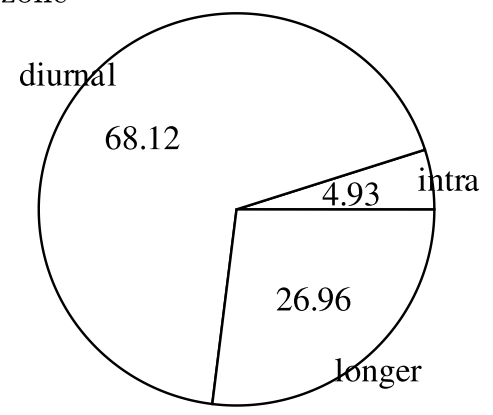

(b) $\mathrm{NO} 2$

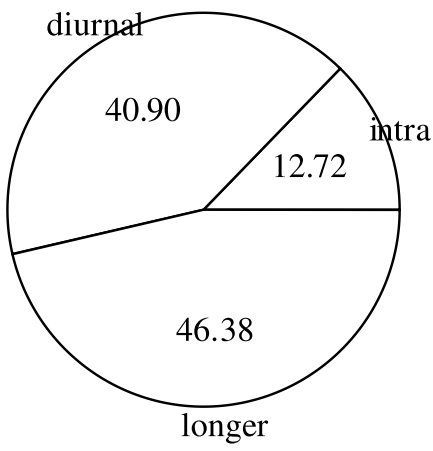

Observed

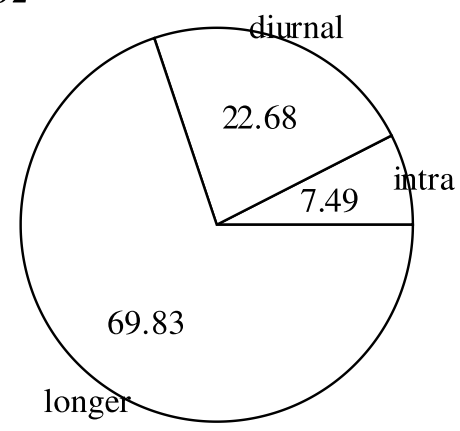

Simulated

(c) $\mathrm{NO}$
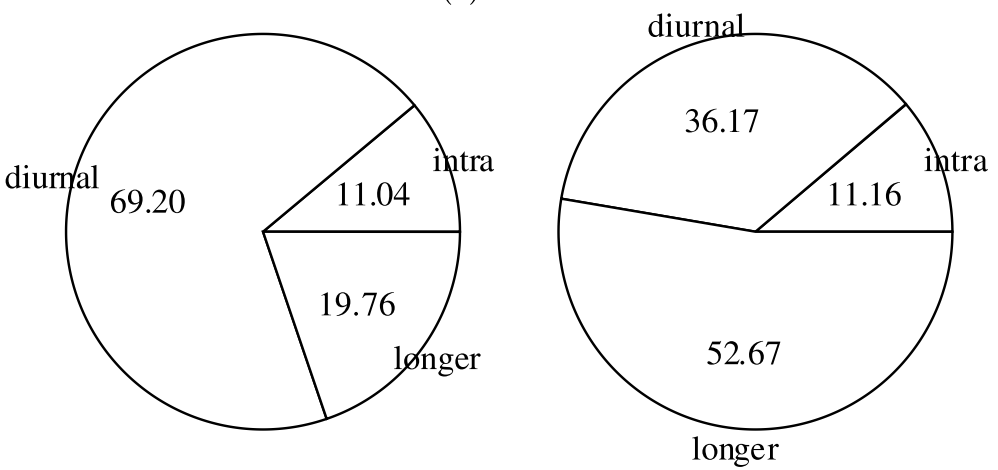

Figure 3: Contribution of different time scales to the total variance of (a) ozone, (b) $\mathrm{NO}_{2}$ and (c) NO. The values are shown as percentage of contribution of time scale component to the total variance. 


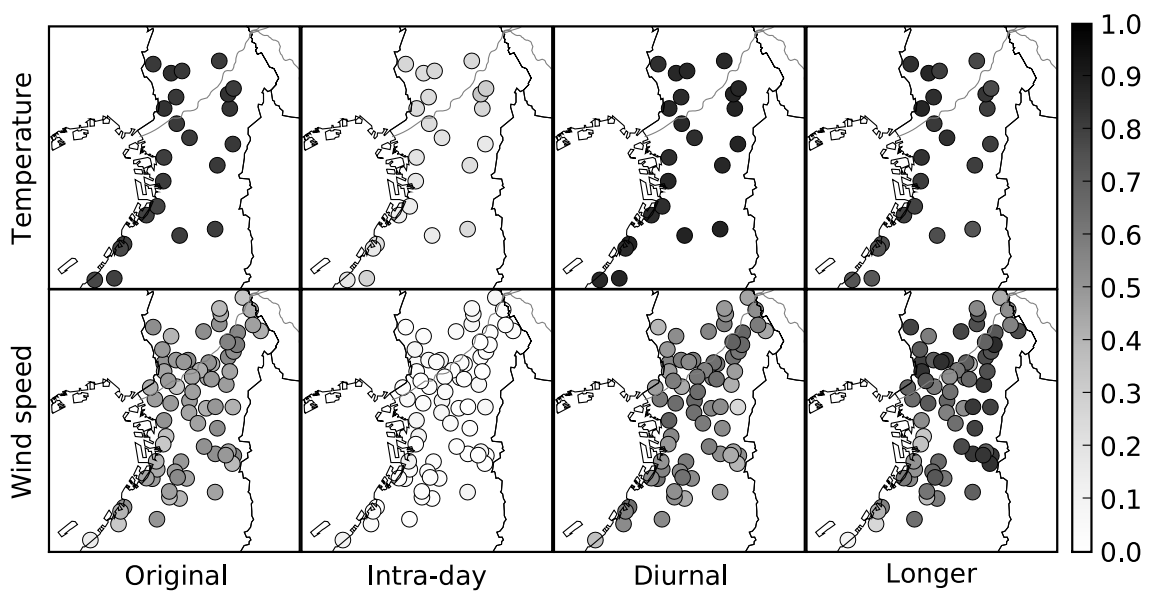

Figure 4: Correlation between simulated and observed temperature and wind speed for different time scales. The light gray line from the top right corner is Yodo River.

prediction in the longer-term component. Though the diurnal processes seem to be the major contributors in determining the time series of $\mathrm{NO}_{2}$ and $\mathrm{NO}$, the longerterm component is apparently contributing the most $(69.83 \%$ and $52.67 \%$ respectively for observed $\mathrm{NO}_{2}$ and NO). This apparently suggests the prevalence of synoptic pattern in $\mathrm{NO}_{x}$ that may have been caused by the unique and complex terrain and geography of the region as well as the lack of sufficient sensitivity to diurnal fluctuations in the observed data.

The correlation coefficient between the simulated and the observed time scale components is helpful in identifying the spatial information of the model performance. The correlation coefficients for near-surface temperature (Fig. 4) are consistently high over 0.8 over all the observation stations of Osaka. Diurnal component is the best simulated time series component with correlation coefficients higher than 0.9 . The longer-term component has also high values $(>0.7)$ but the intra-day component has small correlation coefficients less than 0.3 , especially in the stations near the west coast. The correlation coefficients for wind speed (Fig. 4) are very small in all the stations $(<0.2)$ and the longer-term component has better coefficient values among the time series components. This result is typical of summertime wind, which is affected by model representation of the boundary layer mixing processes and PBL growth and transition periods [13]. The diurnal component of wind speed shows some relatively higher correlation coefficients $(0.6$ to 0.7$)$ in the stations around the Yodo River, which, among other reasons, may be due to complex land use pattern in the region.

Similarly, the correlation coefficients for ozone (Fig. 5) for the diurnal component have higher values $(0.6-0.8)$ in the northern and southern parts of Osaka. The central city and the eastern regions have relatively smaller correlation coefficients (0.5-0.6) for the diurnal component. On the other hand, the longer-term compo- 


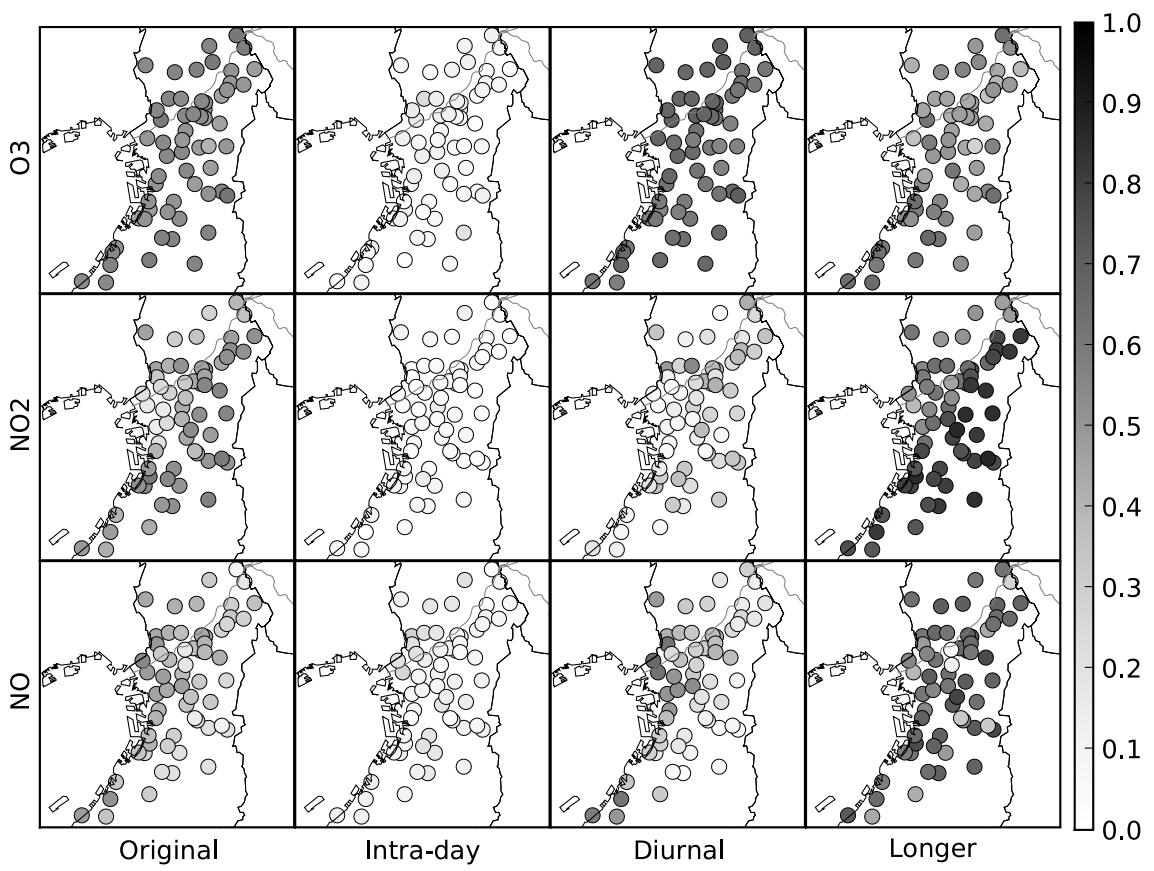

Figure 5: Correlation between simulated and observed ozone, $\mathrm{NO}_{2}$ and $\mathrm{NO}$ for different time scales. The light gray line from the top right corner is Yodo River.

nent has coefficients less than the diurnal component but contains some higher values (around 0.6) along southern Osaka. From these results the higher correlation coefficients for peak ozone concentrations (not shown here) can be explained by the higher correlations in the diurnal and longer-term components. Similarly, the poor correlation in the intra-day component as well as the larger ratio of variability in intra-day time scale (Fig. 3) contributes to the overall gross error.

The correlation coefficients for $\mathrm{NO}_{2}$ and $\mathrm{NO}$ (Fig. 5) have also very small values similar to that of ozone but the overall effect of the components of time scales is spatially different for both of them. The correlation coefficients for $\mathrm{NO}_{2}$ are very low $(<0.2)$ in the downstream region of Yodo River consisting of highly urbanized area, which may have been caused by relatively lower values for the diurnal and longer-term components of $\mathrm{NO}_{2}$. The longer-term component of $\mathrm{NO}_{2}$ has very high correlation coefficients (0.7-0.9) in almost all stations.

In the case of NO, the downstream region of Yodo River has higher overall correlation coefficients (0.4-0.5) as compared to other regions, which may be attributed to relatively higher values for the diurnal component of NO. In both the cases of $\mathrm{NO}_{2}$ and $\mathrm{NO}$, longer-term components show higher correlation coefficients than the other components though the overall correlation coefficients for $\mathrm{NO}$ are comparatively lower than $\mathrm{NO}_{2}$. 


\section{Summary}

High resolution GPV-MSM meteorological data was used for driving the nested domains having 9-km, 3-km and 1-km grid sizes in MM5, and then CMAQ model was run using the meteorological input from MM5 and emission data based on Japanese emission database. Then the time scale components of the meteorological fields (temperature and wind), ozone and NOx were evaluated against the observation data in the Osaka prefecture region using the MM5-CMAQ system.

The time scale analysis reveals a strong influence of spatial distribution on both the meteorological and air quality model outputs. Moreover, the intra-day scales for wind, ozone and $\mathrm{NO}_{x}$ are relatively less well-represented than the other time scales by the MM5-CMAQ system, and thus, a more accurate represented land use and vegetation can be recommended for the terrains of Osaka.

The contribution of intra-day component to the overall variability of air temperature was found to be well simulated though correlation coefficient was low $(<0.4)$ compared to the best simulated diurnal component. Overall, the ozone predictions show the largest contributions from the diurnal component and the longer-term component was the best-simulated time scale for $\mathrm{NO}_{x}$. Similarly, the meteorological fields were also simulated with an adequate level of accuracy.

\section{References}

[1] Sebald, L., Treffeisen, R., Reimer, E. \& Hies, T., Spectral analysis of air pollutants. Part 2: ozone time series. Atmospheric Environment, 34(21), pp. 3503-3509, 2000.

[2] Hogrefe, C., Rao, S., Kasibhatla, P., Hao, W., Sistla, G., Mathur, R. \& McHenry, J., Evaluating the performance of regional-scale photochemical modeling systems: Part II - Ozone predictions. Atmospheric Environment, 35(24), pp. 4175-4188, 2001.

[3] Rao, S.T., Zurbenko, I.G., Neagu, R., Porter, P.S., Ku, J.Y. \& Henry, R.F., Space and time scales in ambient ozone data. Bulletin of the American Meteorological Society, 78(10), pp. 2153-2166, 1997.

[4] Eskridge, R.E., Ku, J.Y., Rao, S.T., Porter, P.S. \& Zurbenko, I.G., Separating different scales of motion in time series of meteorological variables. Bulletin of the American Meteorological Society, 78(7), pp. 1473-1483, 1997.

[5] Rao, S., Ku, J., Berman, S., Zhang, K. \& Mao, H., Summertime characteristics of the atmospheric boundary layer and relationships to ozone levels over the eastern united states. Pure and Applied Geophysics, 160(1), pp. 21-55, 2003.

[6] Dudhia, J., Gill, D., Manning, K., Wang, W. \& Bruyere, C., PSU/NCAR Mesoscale Modeling System Tutorial class notes and users' guide: MM5 Modeling System Version 3, 2005.

[7] Grell, G.A., Dudhia, J. \& Stauffer, D.R., A description of the fifthgeneration Penn State/NCAR mesoscale model (MM5). NCAR Technical Note NCAR/TN-398+STR, 1994. 
[8] Hong, S.Y. \& Pan, H.L., Nonlocal boundary layer vertical diffusion in a medium-range forecast model. Monthly Weather Review, 124(10), pp. 23222339, 1996.

[9] Dudhia, J., A multi-layer soil temperature model for MM5. Preprints, The Sixth PSU/NCAR Mesoscale Model Users’ Workshop, 1996.

[10] Byun, D.W. \& Ching, J.K.S., (eds.) Science Algorithms of the EPA Models3 Community Multiscale Air Quality Model (CMAQ) modeling system. US Environmental Protection Agency, Office of Research and Development, Washington, DC, 1999.

[11] Hogrefe, C., Rao, S., Kasibhatla, P., Kallos, G., Tremback, C., Hao, W., Olerud, D., Xiu, A., McHenry, J. \& Alapaty, K., Evaluating the performance of regional-scale photochemical modeling systems: Part I - Meteorological predictions. Atmospheric Environment, 35(24), pp. 4159-4174, 2001.

[12] Ohsawa, T., Hashimoto, A., Shimada, S., Yoshino, J., de Paus, T., Heinemann, D. \& Lange, B., Evaluation of offshore wind simulations with MM5 in the Japanese and Danish coastal waters. Proceedings of European Wind Energy Conference EWEC: Milan, 2007.

[13] Gilliam, R.C., Hogrefe, C. \& Rao, S., New methods for evaluating meteorological models used in air quality applications. Atmospheric Environment, 40(26), pp. 5073-5086, 2006. 